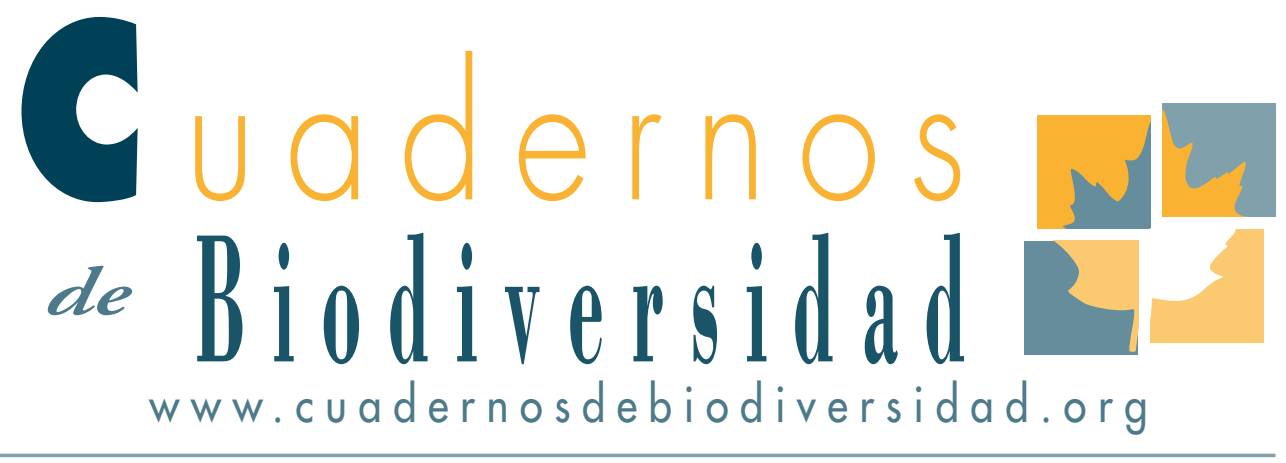

\title{
La utilización de los insectos en la gastronomía, un taller nutritivo
}

\section{Cinta Quirce, Valentina Filippini y Estefanía Micó}

CibiO, Universidad de Alicante. Ctra. San Vicente del Raspeig s/n, 03690, Alicante, España. CINTAQUIRCE@GMAIL.COM

En la tercera edición del curso "Investigando la biodiversidad: presente y futuro" organizado por el CIBIO (Universidad de Alicante), se ha querido dar un enfoque aun más práctico a través de diferentes talleres en los que los alumnos han podido aprender y participar de manera activa mediante actividades como: la edición digital de fotografía de la naturaleza, la historia y elaboración de herberos y la utilización de los insectos en la gastronomía.

Sobre este último taller versa este artículo que resume las actividades realizadas durante el mismo después de que en la primera parte del taller se impartieran conocimientos de distinta índole sobre esta actividad.

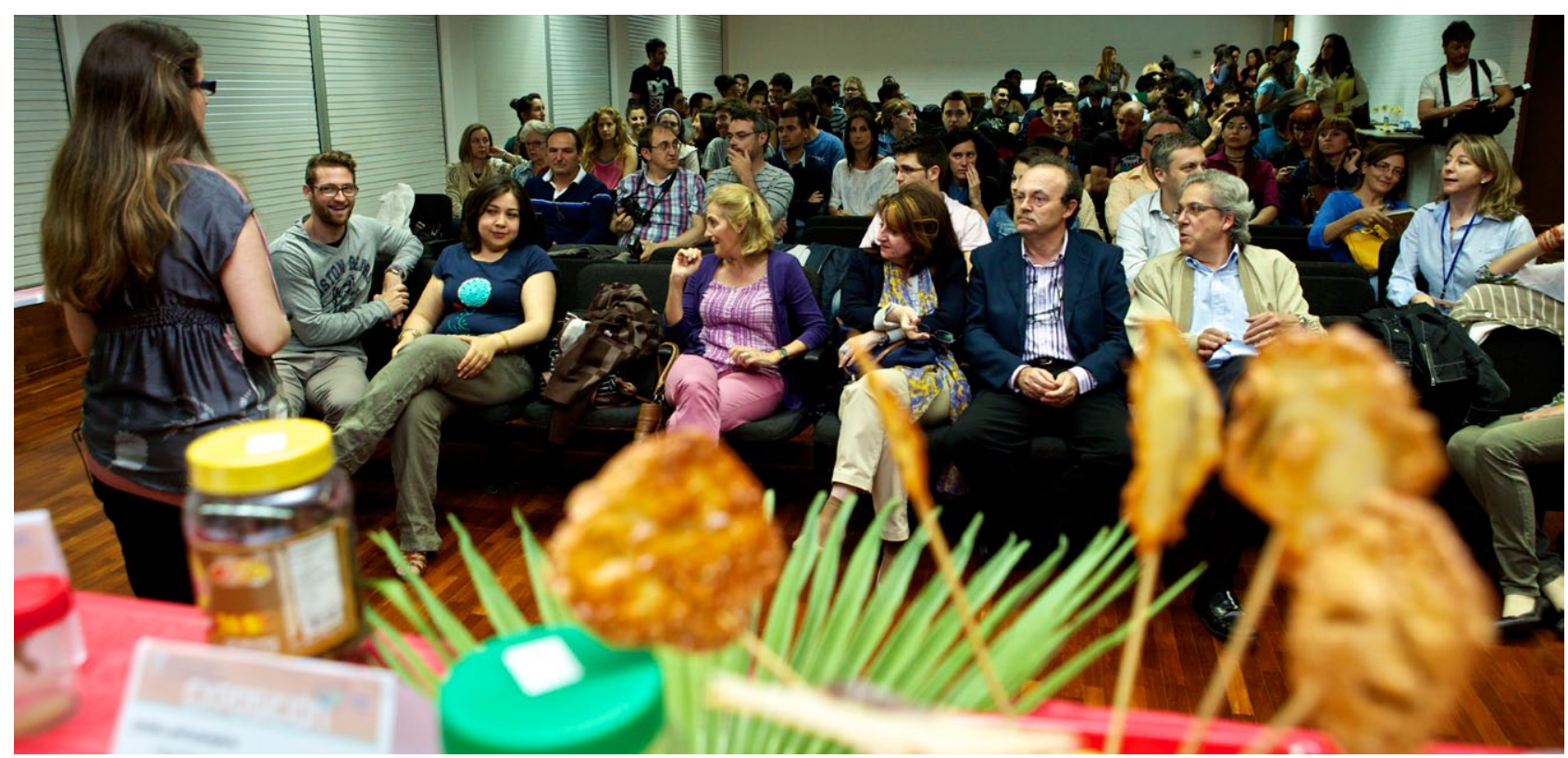




\section{COMER INSECTOS ¿POR QUÉ NO?}

Una manera de acercarse al conocimiento de la biodiversidad animal y vegetal es a través de algo tan cotidiano como el comer. Pero antes de echarnos nada a la boca hay que presentar a los protagonistas, que como veremos dominan el planeta en especies y en número.

El término Entomofagia hace referencia a la ingesta de insectos (Insecta) pero también se amplia a otros grupos de artrópodos (Quelicerados, Miriápodos y Crustáceos) entre los que destacamos a los arácnidos (Chelicerata: Arachnida).

$\mathrm{Al}$ igual que otros artrópodos, la principal característica anatómica de los insectos es la presencia de un exoesqueleto compuesto fundamentalmente de quitina. La quitina es un polisacárido sin ramificar de aminoazúcar de elevado peso molecular, constituido por moléculas de $\mathrm{N}$-acetil-D-glucosamina. Los grupos de moléculas de quitina se organizan en microfibrillas que están incrustadas al lado de las proteínas formando láminas cuya disposición produce una gran fuerza tensora. La presencia de esta peculiar "armadura" junto a otras características morfológicas y de su biología, han convertido a los insectos en un grupo con un éxito evolutivo sin precedentes del que se conocen más de un millón de especies y se estima que el número de especies real podría multiplicar por cinco, como mínimo, esta cifra.

Como sabemos del análisis químico, "la carne" de los insectos, se compone, igual que la de otros animales, de numerosos nutrientes como aminoácidos esenciales, vitamina B12, riboflavina, y varios minerales (como el hierro y el zinc), siendo particularmente rica en proteínas, al mismo nivel que el ganado vacuno y la leche, y también en ácidos grasos insaturados.

Si nos fijamos en su dieta, que es uno de los criterios más comunes para decidir si un animal es o no adecuado para la alimentación humana, vemos que una gran proporción de los insectos dependen íntegramente de materia vegetal, por lo que la mayor parte de las especies que se usan en la gastronomía, son vegetarianos estrictos. Tal y como dice Holt en en una obra publicada por el British Museum en 1885 y que ha sido traducida a muchos idiomas (ver Holt 1997) ¿Cómo es posible que cualquiera que haya comido alguna vez una ostra viva (de hábitos filtradores) le haga ascos a otro animal de hábitos mucho más limpios?, ¿Es más repulsivo un saltamontes, con una dieta vegetariana, que una langosta carroñera?

Desde los tiempos de Homero las cigarras han sido un tema de inspiración para los poetas griegos tanto por su musicalidad como por su delicado sabor. Según Plinio los gourmets romanos tenían la costumbre de engordar para la mesa las larvas de "cossus" dándoles de comer harina y vino. No está clara la identidad del insecto al que se aplicaba el nombre de "cossus" pero se piensa que eran larvas del coleóptero Lucanus cervus (Lucanidae) o de grandes cerambícidos (Cerambycidae) (Holt 1997)

Aunque hoy más de un tercio de la población mundial se alimenta de insectos (Huis et al., 2013), el reparo generalizado a la dieta de insectos parece haber aumentado en el mundo occidental a medida que nos hemos ido distanciando del mundo natural y dejado de estar habituados a ellos.

Teniendo todo esto en cuenta, ¿por qué comer insectos?

Hay poderosos motivos, pero el más intuitivo es que en la actualidad no debemos despreciar ninguna fuente de alimentación saludable. La FAO nos dice que el planeta albergará hasta 9000 millones de personas en 2050 por lo que la producción de alimentos debería duplicarse, pero como veremos a continuación los recursos no son ilimitados. Y en este sentido la cría de insectos podría ser una importante fuente de proteína animal. De la misma forma, el numero de personas con sobrepeso en el mundo occidental supera ya los 1.500 millones de personas, por lo que introducir en la dieta humana fuentes de alimentación más saludables, sería deseable.



Figura 2. Grillos philadelphia, uno de los platos que se degustaron (foto: Roberto Ruiz - Taller de Imagen UA) 


\section{ENTOMOFAGIA COMO PRÁCTICA SOSTENIBLE}

Un aspecto desconocido para la mayoría de los consumidores es el elevado impacto ambiental que ocasiona la ganadería destinada a la producción de carne y leche. Los productos animales a nivel mundial aportan el 30\% de las proteínas totales en la dieta, pero este porcentaje cambia según la zona geográfica: en los países en desarrollo baja a un 26\% mientras que en los países industrializados asciende al 56\%. Esta tendencia de aumentar el consumo de productos animales se va extendiendo hasta los países de economía emergente que intentan imitar el modelo de consumo de estos productos por países desarrollados, por lo que el consumo de carne aumenta más rápidamente que el crecimiento de la población (Premalatha et al. 2011, Steinfeld et al. 2006).

Esto nos debe hacer reflexionar no sólo de si es posible abastecer esta demanda creciente, sino también sobre los efectos que tendría sobre los recursos naturales y el medio ambiente. La ganadería (incluyendo ganado vacuno, porcino, ovino) ocupa el $70 \%$ de las tierras agrícolas, que corresponde al $30 \%$ de la superficie terrestre. Para alimentarlos, destinamos el 34\% de las cosechas de cereales y el $20 \%$ de las capturas de pescado. En total destinamos a la alimentación de ganado 77 millones de toneladas de proteínas, de las que sólo recuperaremos 58 para nuestro consumo (Premalatha et al. 2011). Además, el ganado es responsable del $18 \%$ de todas las emisiones de gases de efecto invernadero de origen antrópico, contando las producidas por los mismos animales y las que provienen de las actividades relacionadas; es un valor comparable a las emisiones del sector transporte (Steinfeld et al. 2006). También liberan otros contaminantes en el suelo y agua: en Estados Unidos la ganadería es responsable del 64\% de la contaminación por amonio, que produce lluvias ácidas, el 50\% de contaminación por antibióticos, el $37 \%$ de fitosanitarios, el 33\% de fósforo y nitrógeno sin contar con los fertilizantes y hormonas que se liberan. Otro impacto es la degradación de los pastos por sobreexplotación, compactación y erosión del suelo, por lo que actualmente el $20 \%$ de los pastos a nivel mundial están degradados (Steinfeld et al.
2006). En cuanto a su impacto sobre la biodiversidad, a parte de los efectos indirectos por los aspectos anteriormente presentados, hay que añadir que la ganadería es la causa del 70\% de la deforestación de bosques tropicales en Sur América, que son reconocidos puntos calientes de biodiversidad (Steinfeld $e t$ al. 2006).

Tomando en cuenta todos los datos presentados hasta ahora, es evidente que la ganadería no es una práctica sostenible en la actualidad. En 2006 la FAO elaboró un informe sobre los efectos negativos de la ganadería sobre el medioambiente, reconociendo esta práctica como una de las principales causas de los problemas ambientales de hoy, y manifestando la necesidad de resolver este problema con urgencia (Steinfeld et al. 2006).

Precisamente uno de los aspectos clave de la entomofagia como fuente principal de proteínas reside en la mayor sostenibilidad de esta práctica respecto a la ganadería tradicional. En cuanto a uso del suelo, los insectos son claramente mucho más pequeños, y pueden ser criados en mayores densidades. Esto permite aprovechar el espacio también en vertical, por lo que se pueden producir $64 \mathrm{~kg}$ de insectos por metro cúbico, frente a los $40 \mathrm{~kg}$ por metro cuadrado de carne de pollo o los $0.13 \mathrm{~kg}$ por metro cuadrado de carne de vacuno (Dzamba 2009).

A su vez, los insectos pueden ser alimentados con partes vegetales desechadas de la dieta humana, como tallos y hojas, o plantas naturales no aprovechadas por el hombre (DeFoliart 2005). Por ejemplo el picudo de México Metamasius spinolae (Coleoptera: Curculionidae) se alimenta de los nopales (Opuntia spp.) que crecen naturalmente en las zonas áridas, y contiene un $69.05 \%$ de proteínas, respecto al $5.21 \%$ de su planta huésped (DeFoliart 1992). Esto permite que no haya competencia para la alimentación entre insectos y seres humanos, y de esta manera no habría que destinar parte de nuestras cosechas a alimentar a nuestras fuentes de alimentos.

Los insectos son mucho más eficaces a la hora de convertir la comida en masa corporal ya que al ser la mayoría de ellos poiquilotermos, no gastan parte de la energía que ingieren en mantener su calor corporal, como aves y mamíferos. Por esto los insectos son cinco veces más eficientes que el ganado vacuno en la conversión de energía, y si consideramos que el 
porcentaje de materia comestible del total del animal es más alto en los insectos que en el ganado $(80 \%$ frente al $40 \%$ del de vacuno), la eficacia asciende a 12 veces (Van Huis et al. 2013).

En cuanto a gases de efecto invernadero, los producidos por los insectos son una centésima parte de los producidos por el ganado, a paridad de peso (Oonincx et al. 2010). Hay que mencionar que algunos insectos como las cucarachas, algunos escarabajos y las termitas, producen metano, que es uno de los gases de efecto invernadero más importante por tener un efecto 23 veces más fuerte que el dióxido de carbono. Esto se debe a su dieta, rica en celulosa, que necesita de una rica fauna microbiana en sus intestinos para poder ser digerida, y que es la responsable de la producción de metano. Estas especies no se están considerando para la cría masiva con fines alimentarios (Oonincx et al. 2010).

En general, podemos afirmar que la huella ecológica de los insectos es la décima parte de la del ganado, a igualdad de peso de producto (Van Huis et al. 2013).

Y ¿qué impacto tendría la difusión de la entomofagia sobre la biodiversidad? La mayoría de los insectos que se consumen actualmente se toman directamente de la naturaleza. Las especies plaga de cultivos, son fácilmente recolectadas y además su uso reduce el nivel de daño que éstas hacen en los cultivos. Por otra parte en la explotación de especies que encontramos en el ámbito natural o seminatural, el conocimiento de los ciclos y de los hábitats de estas especies permite colectarlas con relativa facilidad minimizando el impacto en el medio. Por ejemplo en las zonas de África donde se colectan y consumen varias especies de orugas (larvas de Lepidoptera), la estación de colecta está bien definida, y los recolectores son capaces de regular todo el calendario de otras operaciones de manejo del hábitat como por ejemplo la quema del suelo para fertilizarlo, de esta manera no afecta al ciclo de vida de las larvas y de las demás especies que conviven con ellas (DeFoliart 2005).

Sin embargo, algunas prácticas de colecta dañan de manera puntual al entorno natural, como por ejemplo la búsqueda de nidos de avispas en África, que generan cortes de ramas o del árbol entero, o la recolecta de orugas de Satúrnidos (Nudaurelia oyemensi ) (Lepidoptera) sobre el sapele (Entandro- phragma angolense), una especie arbórea de África tropical que puede llegar a más de $40 \mathrm{~m}$ de altura y a veces es cortado para acelerar la recolecta. Si aumenta considerablemente la demanda de estas especies de insectos comestibles, estas prácticas podrían extenderse, con efectos negativos sobre el entorno (Schabel 2010; Van Huis et al. 2013).

Por ejemplo, en México se ha acusado una clara disminución de algunas especies típicas en la gastronomía, como los escamoles (huevos de la hormiga Liometopum apiculatum) y los gusanos de maguey (orugas de Lepidoptera Aegiale hesperiaris y Xyleutes redtembacheri). Esto se debe a que la subida de la demanda y del precio de estos productos ha causado la aparición de recolectores no tradicionales que ignoran el ciclo de vida y el manejo tradicional de estas especies y además, para intentar generar la máxima ganancia utilizan métodos destructivos. Por ejemplo en el caso de los escamoles, el manejo tradicional prevé dejar un número adecuado de huevos y obreras, para que el nido pueda recuperarse y así seguir siendo productivo. De este modo se puede explotar el mismo nido hasta 3 veces al año durante 30 años. Sin embargo las prácticas recientes destruyen completamente el nido, dejando un número insuficiente de obreras para que el nido se pueda recuperar. En el caso del gusano del maguey, hay que colectar las larvas más grandes, y dejar las larvas más pequeñas, para que puedan completar su ciclo, o dejar plantas intactas, para poder tener la próxima generación de adultos. Pero la sobreexplotación está disminuyendo el número de larvas que llegan a completar el desarrollo, y así el número de adultos (Ramos Elorduy 2006).

Una alternativa es la creación de granjas de insectos, para obtener grandes cantidades sin necesidad de expoliar el entorno natural. Tailandia es uno de los países donde más se está expandiendo esta práctica, gracias al apoyo de la FAO, en forma de instalaciones gestionadas a nivel familiar y de tecnología simple, que sin embargo son altamente efectivas (Johnson 2010).

En Europa, existen diferentes empresas que se dedican a la cría de insectos comestibles a nivel industrial. Este tipo de prácticas están emergiendo a pequeña escala, pero existen aún muchos problemas por solventar: las técnicas de cría se adaptan de 
las empresas de control biológico o de comida para mascotas, sin embargo todavía no hay nada parecido a una selección artificial para mejorar la producción, aspecto que para los demás animales para consumo humano, se está llevando a cabo desde hace milenios. Además se conoce muy poco de los patógenos que podrían presentar los insectos, o enfermedades que podrían afectar a los insectos criados en grandes cantidades. Tampoco se ha profundizado en el estudio del impacto que puede tener la introducción de especies foráneas para cría en áreas donde esas especies no estén presentes (Meyer-Rochow 2010; Van Huis et al. 2013).

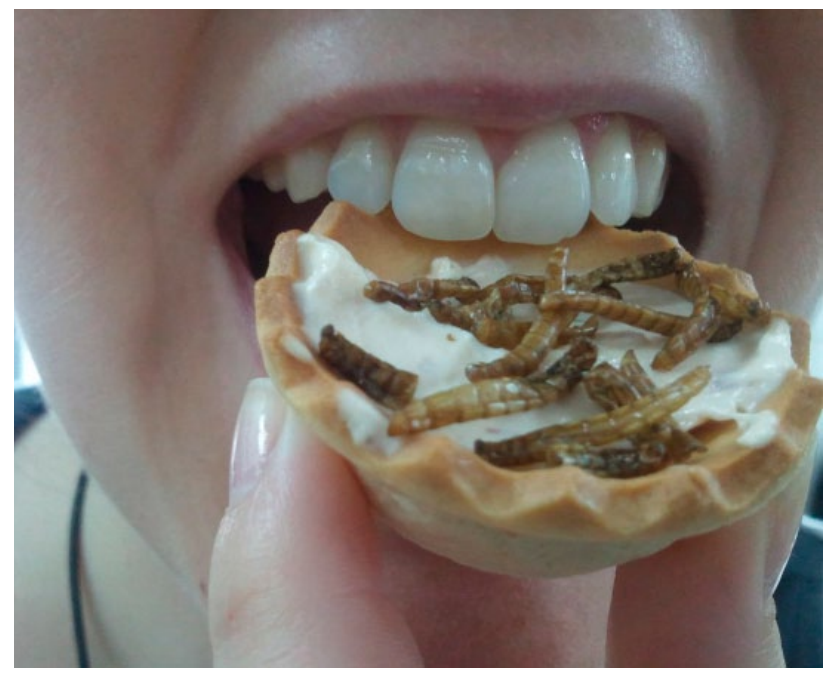

Figura 3. Degustación de tenebriónidos a las finas hierbas (foto: Ma Ángeles Marcos García)

\section{VALORES NUTRICIONALES DE LOS INSECTOS}

En general se consumen los estadios inmaduros de los insectos por tener el cuerpo menos esclerotizado y ser más nutritivos por contener un mayor contenido de grasa. Las propiedades nutricionales varían mucho entre las especies de un mismo orden, y probablemente esté relacionado con la dieta que tienen estos insectos en la naturaleza.

La tabla 1 presenta el porcentaje de proteínas respecto al peso corporal; éste es en general muy

\begin{tabular}{l|c|c}
\hline Grupo & $\mathbf{\%} / \mathbf{1 0 0 g}$ & estado comestible \\
\hline Odonata & $54-56$ & ninfas \\
\hline Ephemeroptera & 58 & ninfas \\
\hline Orthoptera & $52-77$ & ninfas y adultos \\
\hline Hemiptera & $34-70$ & ninfas y adultos \\
\hline Homoptera & 59 & ninfas y adultos \\
\hline Coleoptera & $20-71$ & larvas \\
\hline Lepidoptera & $13-71$ & larvas \\
\hline Diptera & $35-61$ & larvas \\
\hline Hymenoptera & $09-64$ & larvas, pupas, \\
\hline Carne de & $25-28$ & \\
vacuno & & \\
\hline Carne de cerdo & $29-37$ & \\
\hline Pescado & $14-63$ & \\
\hline Alubias & $21-23$ & \\
\hline Soja & $5-35$ & \\
\hline
\end{tabular}

Tabla 1: Porcentaje de proteínas por $100 \mathrm{~g}$ de peso seco (Ramos Elorduy et al 1984).

Los valores para alimentos tradicionales se han consultado en BEDCA.

alto en todos los insectos, con una media mayor a la de la carne vacuna y porcina, o de vegetales ricos en proteínas como frijoles y soja. Muchas especies se acercan además a los valores del pescado, actualmente el alimento más proteico de nuestra dieta.

Además las proteínas proporcionadas por los insectos son de buena calidad, es decir que contienen aminoácidos esenciales, que son los que nuestro organismo no es capaz de sintetizar y que tiene que recibir de la dieta, en proporciones aptas a las necesidades humanas (Bukkens 2005).

Es curioso ver como en muchas comunidades los insectos consumidos tradicionalmente complementan perfectamente la dieta aportando algunos aminoácidos esenciales que están presentes en cantidades limitadas en la comida básica de esas zonas. En Zaire el alimento básico son los cereales, carentes en 
lisina, y las orugas que se consumen habitualmente son una buena fuente de este aminoácido; en Papúa Nueva Guinea, el alimento básico son los tubérculos, limitados en lisina y leucina, y las larvas de los picudos aportan estos dos aminoácidos, y por último, en diferentes zonas de África donde se consume el maíz como alimento principal, pobre en lisina y triptófano, son las termitas las que complementan la dieta (Bukkens 2005).

En cuanto a la cantidad de grasa y energía, los insectos son muy nutritivos, con valores comparables a la carne de cerdo (Tabla 2).

\begin{tabular}{l|c}
\hline Grupo & Kcal/100g \\
\hline Hemiptera & $328-629$ \\
\hline Lepidoptera & $293-777$ \\
\hline Isoptera & $93-761$ \\
\hline Coleoptera & $282-652$ \\
\hline Odonata & $431-520$ \\
\hline Orthoptera & $336-438$ \\
\hline Homoptera & $394-629$ \\
\hline Diptera & $216-499$ \\
\hline Hymenoptera & $380-561$ \\
\hline Carne vacuno & $164-313$ \\
\hline Carne cerdo & $225-541$ \\
\hline Pescado & $105-236$ \\
\hline Alubias & $80-100$ \\
\hline Soja & 100
\end{tabular}

Tabla 2: Valores energéticos para $100 \mathrm{~g}$ de alimento (Ramos Elorduy 2005, Ramos Elorduy et al. 2008). Los valores para alimentos tradicionales se han consultado en BEDCA.

En general los grupos más calóricos son las termitas y las orugas, pero varía según las especies. Los estadios inmaduros suelen ser los de mayor contenido de grasa porque la almacenan para el momento de la metamorfosis.
Cuando hablamos de grasas hay que diferenciar entre diferentes tipos porque aunque los insectos tengan mucha grasa, y por ello aportan muchas calorías, el contenido de colesterol es en general mucho menor al de la carne tradicional, exceptuando la larva del picudo de las palmeras. Sin embargo, los estudios sobre el contenido de colesterol en insectos son muy escasos, comparados con los de contenido proteico o de calorías.

\begin{tabular}{l|c} 
Especie & $\mathbf{m g} / \mathbf{1 0 0} \mathbf{g}$ \\
\hline $\begin{array}{l}\text { Rhynchophorus palmarum } \\
\text { (picudo) (Coleoptera: Curculio- } \\
\text { nidae) }\end{array}$ & 188 \\
\hline Termitas (Isoptera: Termitidae) & $51-61$ \\
\hline $\begin{array}{l}\text { Escarabajos (Coleoptera: Rute- } \\
\text { lidae) }\end{array}$ & 9.1 \\
\hline $\begin{array}{l}\text { Sphingidae (polillas) (Lepidop- } \\
\text { tera) }\end{array}$ & 18 \\
\hline Vacuno & $84-121$ \\
\hline Cerdo & $86-110$
\end{tabular}

Tabla 3: Miligramos de colesterol por $100 \mathrm{~g}$ de producto (Bukkens 2005).

Los valores para alimentos tradicionales se han consultado en BEDCA.

Además, las grasas se pueden diferenciar en grasas saturadas (SFA), monoinsaturadas (MUFA) y poliinsaturadas (PUFA), siendo las saturadas dañinas para la salud cuando se consumen en grandes cantidades. En una dieta balanceada, las grasas saturadas no deberían superar un tercio de todas las grasas en la dieta, mientras que las poliinsaturadas como mínimo deberían representar el 24\%. Como se observa en la tabla 4, la carne tradicional supera los valores de SFA y tiene muy pocos PUFA. En los insectos, aunque los valores de SFA siguen elevados, tienen mucho más PUFA. 


\begin{tabular}{l|c|c|c} 
& Saturadas (SFA \%) & Monoinsaturadas (MUFA \%) & Poliinsaturadas (PUFA \%) \\
\hline Lepidoptera & $32.5-46.5$ & $6.2-67.1$ & $0.4-49.5$ \\
\hline Coleoptera & $42.3-44.3$ & $48.7-66.6$ & $6.5-28$ \\
\hline Isoptera & $37.8-46.7$ & $14.9-51.4$ & $10.9-38.3$ \\
\hline vacuno & $30-50$ & $35-60$ & $1.5-4$ \\
\hline cordero & $40-55$ & $35-45$ & $1.5-7$
\end{tabular}

Tabla 4: Porcentajes de grasas saturadas e insaturadas sobre el total de la grasa presente en el alimento (Bukkens 2005).

Los insectos también son fuente de fibra, y eso no debe sorprendernos si pensamos en el exosqueleto de quitina indigerible, con valores que se acercan a alimentos como los cereales $(9.5 \mathrm{~g} / 100 \mathrm{~g})$, mientras que la carne tradicional no tiene fibra. También las orugas $(6.5-11.4 \mathrm{~g} / 100 \mathrm{~g})$, que tienen exosqueleto más blando, pueden tener un contenido de fibra comparable a los de insectos adultos y bien esclerotizados como grillos y saltamontes (4.9-12.1 g/100 g) (Bukkens 2005).

Los insectos son una buena fuente de vitaminas del grupo B y de minerales, $100 \mathrm{~g}$ de varias especies de insectos aportan mucho más de la cantidad diaria recomendada.

\begin{tabular}{l|c|c|c} 
& Tiamina (B1) & Riboflavina (B2) & Niacina (B3) \\
\hline Cantidad diaria recomendada & $1.5 \mathrm{mg}$ & $1.7 \mathrm{mg}$ & $20 \mathrm{mg}$ \\
\hline Pollo & $5.4 \%$ & - & $45 \%$ \\
\hline Frijoles & $10.8 \%$ & - & $3 \%$ \\
\hline Termitas & $8.7 \%$ & $67.4 \%$ & $47.7 \%$ \\
\hline Gusano de seda & $224.7 \%$ & $112.2 \%$ & $26 \%$ \\
\hline Picudo de palmera (Angola) & $201.3 \%$ & $131.7 \%$ & $38.9 \%$ \\
\hline Axalacatl (chinche - México) & & $119 \%$ & \\
\hline Orugas (Angola) & $244.7 \%$ & $112.2 \%$ & $26.0 \%$
\end{tabular}

Tabla 5: Contenido de vitaminas del grupo B expresado como porcentaje de la cantidad diaria recomendada (Bukkens 2005, Premalatha et al. 2011).

\begin{tabular}{l|c|c|c|c|c|c}
\cline { 2 - 6 } & $\mathbf{C a}$ & $\mathbf{P}$ & $\mathbf{M g}$ & $\mathbf{F e}$ & $\mathbf{C u}$ & $\mathbf{Z n}$ \\
\hline Cantidad diaria & $1 \mathrm{~g}$ & $1 \mathrm{~g}$ & $400 \mathrm{mg}$ & $18 \mathrm{mg}$ & $2 \mathrm{mg}$ & $15 \mathrm{mg}$ \\
\hline Carne vacuno & & & & $16 \%$ & & \\
\hline Termitas & $4.0 \%$ & $43.8 \%$ & $104.2 \%$ & $41.7 \%$ & $680 \%$ & - \\
\hline Gusano de seda & $35.5 \%$ & $69.5 \%$ & $13.5 \%$ & $197.2 \%$ & $120 \%$ & $153.3 \%$ \\
\hline Picudo de palmera (Angola) & $18.6 \%$ & $31.4 \%$ & $7.5 \%$ & $72.8 \%$ & $70 \%$ & $158 \%$ \\
\hline Axalacatl (chinche - México) & & & & $267 \%$ & &
\end{tabular}

Tabla 6: Contenido de micronutrientes minerales expresado como porcentajede la cantidad diaria recomendada (Bukkens 2005). 
Las ventajas ofrecidas por los insectos como comida nutritiva y de cría fácil, han hecho que se estén considerando los insectos como comida para astronautas, un proyecto llevado adelante por un grupo de científicos japoneses, que han elaborado una dieta óptima a base de vegetales y gusanos de seda (Katayama et al. 2008).

\section{LOS HUMANOS TAMBIÉN PICAN: INSECTOS COMESTIBLES}

Para aproximadamente 2.000 millones de personas, es decir, para aproximadamente un tercio de la población mundial comer insectos forma parte de su dieta diaria, de manera similar a comer carne o pescado (Van Huis et al. 2013).

Podemos encontrar mucha información sobre la entomofagia: libros de cocina, páginas web, recetas online, proveedores y restaurantes especializados en platos a base de insectos; el cocinero David George Gordon escribe el bestseller Eat-a-Bug Cookbook donde muestra diferentes recetas recogidas alrededor del mundo y nos detalla las que para él tienen mejor sabor. La entomóloga Julieta Ramos Elorduy de la UNAM (Universidad Nacional Autónoma de México) y su equipo de investigación, en estos últimos ańos han producido varias patentes internacionales de cria intensiva de insectos destinados al consumo humano, que además incluyen investigaciones para la selección de individuos, análisis genético y nutricional y otros protocolos de control de calidad, para conseguir un producto final con propiedades óptimas para el consumo.

Actualmente, la FAO impulsa la entomofagia en distintas regiones del mundo; junto a la Universidad Wageningen de Holanda y consultores expertos, realizó en enero de 2012 una reunión para evaluar el potencial de los insectos como alimento y para garantizar la seguridad alimentaria. Entre las conclusiones del evento destacaron que los insectos pueden ser una alternativa para paliar la malnutrición o el hambre, además de una oportunidad de empleo e ingresos para personas de bajos recursos.

Gracias a la investigación de Jongema de la Uni- versidad Wageningen de Holanda hoy sabemos que existen unas 1900 especies diferentes de insectos comestibles en el mundo (Van Huis et al. 2013). El mayor número de países y personas que incluyen a los insectos en su dieta se encuentran en Asia, África y América latina, por el contrario en América del Norte y Europa son las zonas donde la entomofagia es desconocida o casi no se practica. En Estados Unidos la entomofagia empieza a conocerse debido a la influencia de los inmigrantes procedentes, sobretodo, de Asia y México y ya se pueden encontrar restaurantes donde degustar a los insectos.

Cabe resaltar México, un país con una elevada biodiversidad tanto cultural como de riqueza de especies, donde esa biodiversidad también se ve reflejada en el número de especies de insectos y otros artrópodos comestibles y donde diferentes culturas consumen insectos desde hace cientos de años. Por todas estas razones y por la investigación tan exhaustiva en el campo de la entomofagia dirigida por la Dra. Ramos, México es uno de los países donde en la actualidad hay una elevada oferta de especies de insectos que a día de hoy, se estima que podría estar por encima de las 600 especies diferentes. A continuación citaremos algunos nombres conocidos como:

- Los chapulines, que se utilizan para designar a un gran número de especies de saltamontes (Orthoptera) comestibles.

- Los gusanos de maguey, Aegiale hesperiaris y Xyleutes redtembacheri (Lepidoptera), que se alimentan de la planta Agave.

- Los jumiles: también conocidos como chinches de monte o xotlinilli para varias especies de insectos hemípteros comestibles de la familia Pentatomidae (Euschistus taxcoensis, Atizies taxcoensis, entre otros).

- Los escamoles, denominados así los huevos del género de la hormiga Liometopum que son considerados un ingrediente de lujo.

- Las hormigas culonas, que son las reinas de diferentes especies pertenecientes al género $\mathrm{Atta}$, muy conocidas en toda Latinoamérica pero que se consumen principalmente en Venezuela, México y Brasil. 


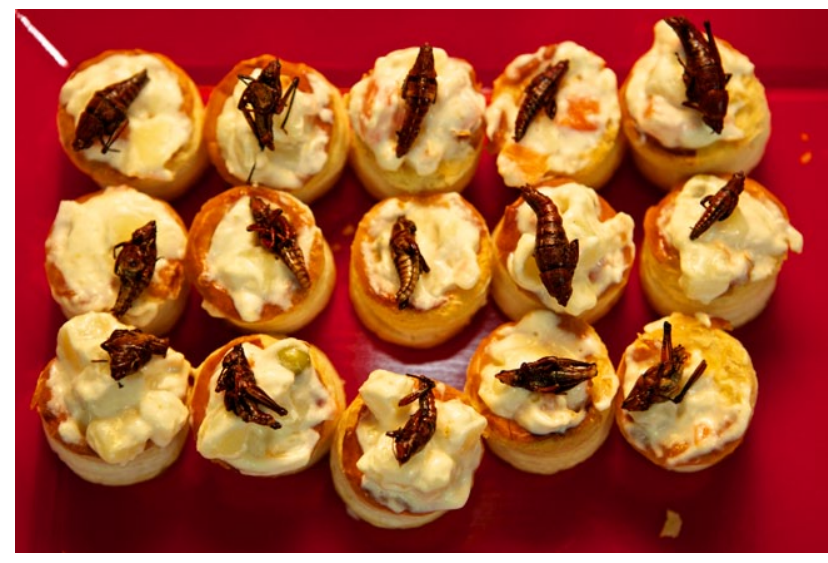

Figura 4. Vou al vent rusos con chapulines. (foto: Roberto Ruiz - Taller de Imagen UA)

En Europa no es común el consumo de insectos, pero en algunos países como Holanda existen diferentes líneas de investigación sobre insectos comestibles y ya existen empresas que comercializan insectos para consumo humano. En Francia se importan insectos para utilizarlos en restaurantes e incorporarlos a la dieta en forma de platos elaborados. En Inglaterra no existe tampoco un claro rechazo a la entomofagia y los insectos se comercializan en forma de snacks, como por ejemplo: "grillos thai al curry", los "gusanos fritos BBQ" o las "hormigas tostadas" que importan sobretodo de Tailandia.

La situación en Espańa no es la misma, en nuestro país ha habido comercios donde podíamos encontrar insectos para consumo humano y algunos restaurantes que tuvieron la iniciativa de incluir los insectos en sus menús. Pero en todas estas ocasiones por falta de legislación en España sobre la legalidad de la venta o consumo o simplemente por la falta de público interesado, estas ideas no han prosperado. Existen diferentes empresas que se dedican a la cría masiva de insectos, aunque únicamente para la dieta de mascotas exóticas o como harina o pienso para el ganado. Quizás en algún futuro estas empresas puedan reconducir la producción de insectos para el consumo humano y podamos encontrarlos envasados en las estanterías de los supermercados.
El seminario tuvo su sesión práctica que consistió en una degustación con el ingrediente estrella del taller: los insectos. A continuación se detallan los platos que se pudieron degustar durante el taller.

- Grillos philadelphia

- Ingredientes: pan tostado, queso philadelphia y nuestro ingrediente: grillo

- Fantasía de mozarella

- Ingredientes: queso mozarella y larvas de tenebriónido (Coleoptera: Tenebrionidae)

- Tenebriónidos a las finas hierbas

- Ingredientes: pan tostado, queso a las finas hierbas y larvas de tenebriónido (Coleoptera: Tenebrionidae)

\section{- Tartaleta dulce de hormiga}

- Ingredientes: tartaleta, queso mascarpone, membrillo y/o miel y hormigas (Hymenoptera: Formicidae)

\section{- Sabores mexicanos con chapulines}

- Ingredientes: nachos con salsa "pico de gallo" y nachos con salsa tajín (especias mexicanas) con "chapulines" (Orthoptera)

\section{- Saltamontes a la española}

- Ingredientes: pincho de tortilla de patata con saltamontes (Orthoptera)

- Pincho de la huerta

- Ingredientes: Tomate cherry, queso fresco y saltamontes

- Vou al vent rusos

- Ingredientes: Hojaldre, ensaladilla rusa y saltamontes

- Bombones de hormigas

- Ingredientes: chocolate puro, cereales crujientes y hormigas tejedoras

- Chupitos entomológicos

- Ingredientes: Gelatina de limón y saltamontes 


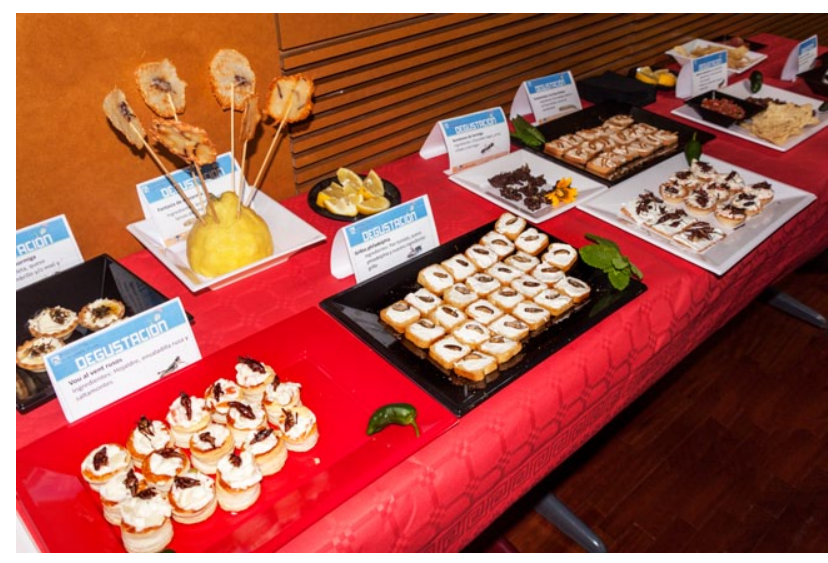

Figura 5. Detalle de una de las mesas del taller con los platos para degustación (foto: Roderic Pla)

Y asados o fritos tuvimos:

- Pupas de gusano de seda (Lepidoptera: Bombycidae: Bombyx mori)

- Piruletas de escorpión (Scorpionida: Scorpionidae: Heterometrus Spinifer)

- Larvas de picudo (Coleoptera: Curculionidae: Rhynchophorus ferrugineus)

- Picudo adulto (Coleoptera: Curculionidae: Rhynchophorus ferrugineus)

- Hormigas: huevos, pupas, reinas, aladas y obreras (Hymenoptera: Formicidae: Oecophylla)

- Alacrán cebollero (Orthoptera: Gryllotalpidae: Gryllotalpa gryllotalpa)

- Grillos (Orthoptera: Gryllidae: Gryllus)

A este taller asistieron más de 100 personas y aunque algunas asistieron con curiosidad y con reticencia a probar alguno de los insectos podemos decir que al final del taller el $90 \%$ dejaron a un lado sus "miedos" y probaron los diferentes platos.

Gracias a las últimas noticias generadas por diferentes informes y trabajos científicos emitidos por la FAO recomendando el consumo de insectos para paliar el hambre en el mundo y como alimento contra la obesidad parece que la entomofagia se está conociendo un poco más y ya no descartamos

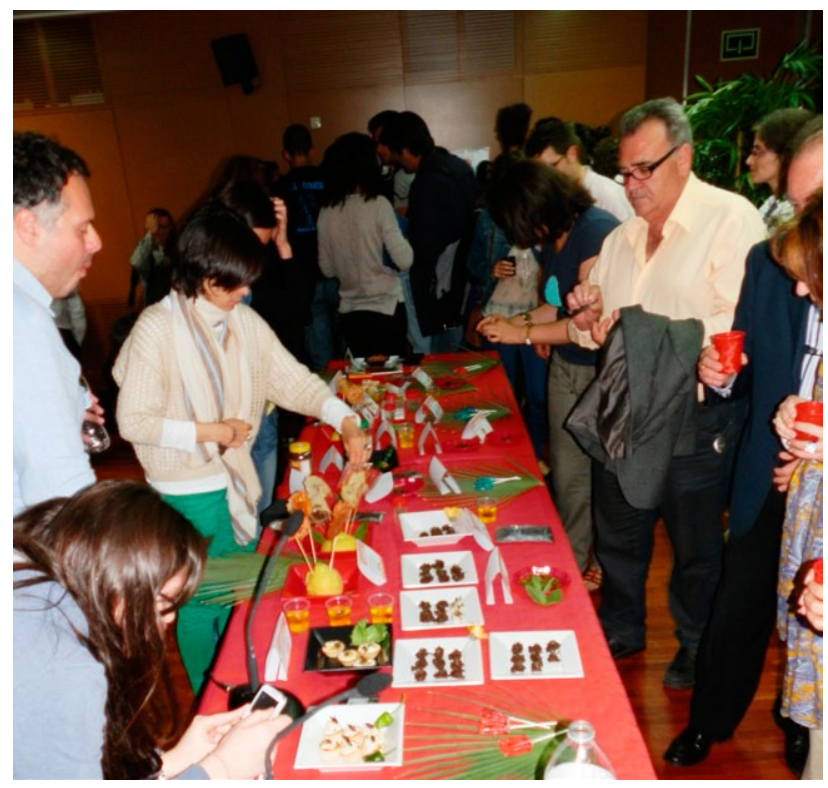

Figura 6. Algunos de los asistentes y platos que degustaron (foto: Alfredo Hernández)

completamente a los insectos como alimento para el ser humano. Es posible que en unos cuantos años nuestra "conciencia" y nuestras leyes nos permitan consumir insectos como un ingrediente más en nuestra dieta.

\section{BIBLIOGRAFÍA}

BEDCA: Base de datos española de composición de alimentos. http://www.bedca.net/

Bukkens, S. 2005. Insects in the human diet: nutritional aspects. In: M. Paoletti (ed). Ecological implications of minilivestock. Science Publishers, Enfield (NH), USA: 546-577.

DeFoliart, G. 1992. Insects as human food. Crop Protection 11(5): 395-399.

DeFoliart, G. 2005. Overview of role of edible insects in preserving biodiversity. In: M. Paoletti (ed). Ecological implications of minilivestock. Science Publishers, Enfield (NH), USA: 123-140.

Dzamba, J. 2009. Third Millenium Farming. http://www. thirdmillenniumfarming.com/01.\%20Online $\% 20$ Files/03.\%203MF\%20Research\%20Brochure.pdf 
Holt, V.M. 1997. ¿Por qué no comer insectos?. En: A. Melic (ed). Los artrópodos y el hombre. Bol. SEA, 20: 249-257

Huis, A. van, Itterbeeck, J. van, Klunder, H., Mertens, E., Halloran, A. Muir, G., Vantomme, P. 2013. Edible Insects: future prospects for food and feed security. FAO Forestry paper 171, Rome: 187pp.

Katayama, N., Ishikawa, Y., Takaoki, M., Yamashita, M., Nakayama, S., Kiguchi, K., Kok, R., Wada, H., Mitsuhashi, J. \& Space Agriculture Task Force. 2008. Entomophagy: A key to space agriculture. Advances in Space Research, 41(5): 701-705.

Johnson, D.V. 2010. The contribution of edible forest insects to human nutrition and to forest management: current status and future potential. In: Durst, P.B., Johnson, D.V., Leslie, R.N. and Shono, K. (eds): Edible Forest Insects: humans bite back. FAO, Regional Office for Asia and the Pacific, Thailand: 5-22.

Meyer-Rochow, V.B. 2010. Entomophagy and its impact on world cultures: the need for a multidisciplinary approach. In: Durst, P.B., Johnson, D.V., Leslie, R.N. and Shono, K. (eds): Edible Forest Insects: humans bite back. FAO, Regional Office for Asia and the Pacific, Thailand: 23-36.

Oonincx, D.G., van Itterbeeck, J., Heetkamp, M.J.W., van den Brand, H., van Loon, J.J.A. and van Huis, A. 2010. An exploration on greenhouse gas and ammonia production by insect species suitable for animal or human consumption. PloS ONE 5(12): 1-7.
Premalatha, M., Abbasi, T., Abbasi, T. and Abbasi S.A. 2011. Energy-efficient food production to reduce global warmind and ecodegradation: the use of edible insects. Renawable and Suistanable Energy Reviews 15: 43574360 .

Ramos Elorduy, J. 2005. Insects: a hopeful food source. In: M. Paoletti (ed). Ecological implications of minilivestock. Science Publishers, Enfield (NH), USA: 263-291

Ramos Elorduy, J. 2006. Threatened edible insects in Hidalgo, Mexico and some measures to preserve them. Journal of Ethnobiology and Ethnomedicine 2: 51.

Ramos Elorduy, J., Pino Moreno, J.M., \& Marquez Mayaudon, C. 1984. Protein content of some edible insects in Mexico. Journal of Ethnobiology 4(1):61-72.

Ramos Elorduy, J., Pino, J.M., \& Cuevas Correa, S. 2008. Insectos comestibles del Estado de México y determinación de su valor nutritivo. Anales del Instituto de Biología de la UNAM, Serie Zoología 69(1):65-104.

Schabel, H.G. 2010. Forest Insects as food: a global review. In: Durst, P.B., Johnson, D.V., Leslie, R.N. and Shono, K. (eds): Edible Forest Insects: humans bite back. FAO, Regional Office for Asia and the Pacific, Thailand: 37-64.

Steinfeld, H., Gerber, P., Wassenaar, T., Castel, V., Rosales, M., and de Haan, C. 2006. Livestock's long shadow. FAO, Roma: 390pp. 